\title{
ASPECTOS CLÍNICO-ECONÔMICOS DA QUIMIOTERAPIA ADJUVANTE NO CÂNCER DE MAMA HER-2 POSITIVO
}

\author{
Sandro José Martins*, Crystina Aoki Yamamoto \\ Trabalho realizado pela Coordenação de Assistência à Saúde (PLANSERV), Secretaria de Administração do Estado da Bahia, Salvador, BA
}

*Correspondência:

Av. Sete de Setembro, 3043

- Apto 301 - Barra

Salvador - Bahia

Cep 40130-000

sandro.martins@gmail.com

\section{RESUMO}

OBjetivo. Avaliar o impacto clínico e os custos do tratamento adjuvante para câncer de mama com superexpressão do receptor 2 do Fator de Crescimento Epidérmico (HER-2).

Métodos. Foram obtidas medidas de eficácia (sobrevida livre de doença em 3 anos) dos ensaios de fase III com trastuzumabe para o câncer de mama HER-2 positivo: um estudo finlandês (FinHER), dois americanos (National Surgical Adjuvant Breast and Bowel Project - NSAPB-3I e North Central Cancer Treatment Group N983I) e dois multinacionais (Herceptin Adjuvant, HERA e Breast Cancer International Research Group, BCIRG-006), e calculadas medidas de impacto clínico: redução de risco e número necessário para tratar (NNT). Foram estimados os custos com medicamentos antineoplásicos nestes diferentes regimes terapêuticos.

Resultados. A redução absoluta de risco com uso do trastuzumabe foi maior no estudo FinHER (II,7\%; IC 95\%: 2,2\% a 21,2\%). O NNT no FinHER foi 8 (IC 95\%: 3 a 28), 8 (IC 95\%: 7 a II) no NSABP-3I/N983I, I2 (IC 95\%: 9 a I I) no HERA, I4 (IC 95\%: II a 24) no BCIRG/com antraciclinas e 17 (IC 95\%: 12 a 34) no BCIRG/sem antraciclinas. O custo para evitar um caso de recidiva seria $R \$ 418.285,44$ com o regime FinHER, $R \$ 1.716 .789,44$ no NSABP-31/N9831, $R \$ 2.481 .891,58$ no HERA, R\$2.963.634,62 no BCIRG/com antraciclinas e $R \$ 3.930 .520,43$ no BCIRG/sem antraciclinas. Conclusäo. Restringir o uso do trastuzumabe à fase inicial de quimioterapia adjuvante (FinHER) permite beneficiar, do ponto de vista econômico, quatro a nove vezes mais pacientes que a monoterapia adjuvante prolongada como usada nos demais protocolos.

UnITERMOS: Análise custo-eficiência. Gastos em saúde. Neoplasias mamárias. Quimioterapia adjuvante. Saúde suplementar. Trastuzumabe.

\section{INTRODUÇÃo}

Cerca de 25\% das mulheres com câncer de mama apresentam superexpressão do receptor 2 para o fator de crescimento epidermal (HER-2/neu), casos estes associados a uma doença de comportamento agressivo, com alta probabilidade de recorrência após o tratamento inicial e portanto um prognóstico mais reservado ', 2.

Na literatura médica há cinco estudos prospectivos de fase III que avaliaram o papel do trastuzumabe, um anticorpo monoclonal bloqueador do receptor HER-2, na quimioterapia adjuvante de mulheres com câncer de mama HER-2 positivo ${ }^{3}$ : um estudo europeu (Finland Herceptin, FinHER), dois estudos americanos (National Surgical Adjuvant Breast and Bowel Project NSAPB-3 I e North Central Cancer Treatment Group N983I) e dois estudos multinacionais (Herceptin Adjuvant, HERA e Breast Cancer International Research Group, BCIRG-006). Todos foram concordantes em demonstrar que o uso deste medicamento promove uma redução importante do risco de recidiva desta variante agressiva de câncer de mama ${ }^{4.8}$.

Nestes ensaios clínicos, os investigadores diferiram quanto à estratégia de incorporação do trastuzumabe ao tratamento convencional. Enquanto o estudo FinHER mostrou benefício e boa segurança para o uso do anticorpo combinado a drogas citotóxicas que interferem com o fuso mitótico (docetaxel ou vinorelbina) nos ciclos iniciais da quimioterapia adjuvante, nos estudos NSABP-B3I, N983I, HERA e BCIRG-006 foi demonstrada vantagem para a monoterapia adjuvante de manutenção por pelo menos um ano com o trastuzumabe, ainda que com toxicidade cardíaca significativa em 4,5\% das pacientes ${ }^{3}$.

Idealmente, a incorporação de uma tecnologia pelo sistema de saúde, público ou privado, deveria ser amparada não apenas em estimativas pontuais de eficácia e no princípio da eqüidade, como também na revisão sistemática, crítica, criteriosa da literatura disponível, considerando-se aspectos como efetividade da intervenção, análise econômica do seu potencial para contribuir na promoção, manutenção ou recuperação da saúde. Nas últimas décadas, a elevação quase universal dos gastos em saúde e os crescentes constrangimentos decorrentes da escassez de recursos vis-à-vis demanda têm imposto a necessidade de se justificar os novos métodos diagnósticos e terapêuticos em termos de seu custo-efetividade?

Em nosso meio há poucos relatos de investigações sobre custos em oncologia, área da medicina que tem experimentado rápida incorporação de terapias de alto custo. Neste trabalho apresentamos uma 
avaliação dos custos com medicamentos de diferentes estratégias terapêuticas para quimioterapia adjuvante em mulheres com câncer de mama HER-2/neu positivo.

\section{Métodos}

Este é um estudo descritivo do impacto clínico e dos custos com medicamentos de diferentes estratégias atuais de quimioterapia adjuvante para o câncer de mama HER-2/neu positivo.

Encontra-se na literatura a descrição de cinco ensaios clínicos que avaliaram o uso do trastuzumabe na quimioterapia adjuvante para o câncer de mama HER2/neu-positivo 3 . Para estes estudos foram eleǵveis mulheres com câncer de mama invasivo e envolvimento linfonodal, ou com doença linfonodo-negativo e de alto-risco para recidiva (FinHER, N9831, HERA, BCIRG 006), julgadas medicamente aptas ao tratamento adjuvante apropriado (quimioterapia, radioterapia e hormonioterapia). Como o trastuzumabe é um medicamento cardiotóxico, a presença de doença cardíaca em várias formas era considerada fator de exclusão.

O FinHER foi o primeiro ensaio clínico a demonstrar benefício para o uso breve do trastuzumabe na quimioterapia adjuvante para o câncer de mama HER-2/neu-positivo4. Delineado para comparar dois agentes citotóxicos na quimioterapia adjuvante do câncer de mama (docetaxel $100 \mathrm{mg} / \mathrm{m} 2$ por três ciclos ou vinorelbina $25 \mathrm{mg} / \mathrm{m} 2 / \mathrm{semanal}$ por nove semanas, seguidos por três ciclos de 5 -fluoruracil $600 \mathrm{mg} / \mathrm{m} 2$, epirrubicina $60 \mathrm{mg} / \mathrm{m} 2$ e ciclofosfamida $600 \mathrm{mg} / \mathrm{m} 2$ ), pacientes com superexpressão HER-2/neu foram assignados para usar ou não trastuzumabe (4 mg/kg inicial e $2 \mathrm{mg} / \mathrm{kg}$ semanal por oito semanas) concomitante a um destes citotóxicos iniciais.

O estudo NSABP B-3 I comparou quatro ciclos de doxorrubicina 60 $\mathrm{mg} / \mathrm{m} 2$ e ciclofosfamida $600 \mathrm{mg} / \mathrm{m} 2$, seguidos por quatro ciclos de paclitaxel $175 \mathrm{mg} / \mathrm{m} 2$ (AC-P) ao mesmo esquema associado ao trastuzumabe $4 \mathrm{mg} / \mathrm{kg}$ inicial e $2 \mathrm{mg} / \mathrm{kg}$ semanal por $5 \mathrm{I}$ semanas (AC-PT). O protocolo N983I comparava dois tratamentos experimentais a um controle semelhante ao usado no NSABP B-3I, mas com administração semanal de paclitaxel $80 \mathrm{mg} / \mathrm{m} 2$. Os tratamentos experimentais variavam quanto ao momento de introdução do trastuzumabe: sequencial à quimioterapia convencional (AC-P-T) ou concomitante aos ciclos com paclitaxel (AC-PT), a maneira usada no protocolo NSABP B-3I . Considerando o lento recrutamento nestes estudos, critérios de elegibilidade similares e os regimes experimentais semelhantes, houve uma emenda aos protocolos para permitir a análise conjunta dos grupos controle (AC-P) e experimental (AC-PT), não sendo incluídos aqueles que receberam trastuzumabe de modo sequencial'.

O estudo HERA foi concebido para avaliar o uso de trastuzumabe por um ou dois anos após quimioterapia neo-adjuvante e/ou adjuvante convencional, sendo o regime citotóxico selecionado por cada Centro participante dentro de padrões aceitáveis de tratamento ${ }^{5}$. Na análise de eficácia mais recente foram revistos os dados de 1.698 mulheres que participavam do grupo controle e 1.703 assignadas para receber trastuzumabe por um $a n o^{8}$.

O estudo BCIRG-006 avaliava a segurança e eficácia de três regimes experimentais contendo docetaxel, um medicamento da mesma classe terapêutica que o paclitaxel e ativo no tratamento do câncer de mama: AC por quatro ciclos seguido por quatro ciclos de docetaxel $100 \mathrm{mg} / \mathrm{m} 2$ (AC-D); AC seguido por docetaxel (quatro ciclos) e trastuzumabe $2 \mathrm{mg} / \mathrm{kg}$ semanal por um ano (AC-DT); e um esquema sem doxorrubicina - carboplatina (área sob concentração pelo tempo de $6 \mathrm{mg} / \mathrm{mL} / \mathrm{min}$ ) e docetaxel $75 \mathrm{mg} / \mathrm{m} 2$ por seis ciclos e tratstuzumabe $2 \mathrm{mg} / \mathrm{kg}$ semanal por um ano (CbDT) .

\section{Medidas de eficácia}

A partir de artigos completos e resumos publicados até outubro de 2007 foram obtidas estimativas das medidas de eficácia dos estudos FinHER, NSABP-3I/N983I, HERA e BCIRG-006, na forma de razão de riscos para sobrevida livre de doença após três anos. Até esta época não estavam disponíveis os dados de eficácia específicos para o estudo NSABP-B3I, pelo que incluímos a análise conjunta de participantes dos protocolos NSABP-B3I e N98316. Estas medidas foram convertidas em estimativas de impacto clínico: redução de risco (relativa e absoluta) e número necessário para tratar para evitar um desfecho (NNT); foram calculados os limites para intervalos de confiança de $95 \%$ para as medidas de impacto clínico ${ }^{10}$.

\section{Custos do tratamento}

A perspectiva de análise de custos utilizada foi a do sistema de saúde suplementar, limitada às despesas com medicamentos antineoplásicos. Não foram incluídas nesta análise, portanto, despesas com insumos e medicamentos de suporte, honorários profissionais, custos fixos ou taxas operacionais aplicáveis à terapia antineoplásica ambulatorial, custos indiretos com absenteísmo, exames específicos para pesquisa da superexpressão de HER-2/neu, avaliação e monitoramento cardíaco, e o acompanhamento e tratamento de efeitos adversos.

Utilizou-se como estimativa de custos com medicamentos os valores de reembolso referentes ao preço máximo ao consumidor, como estabelecido em 10/9/2007 pela Câmara de Regulação do Mercado de Medicamentos da Agência Nacional de Vigilância Sanitária para os seguintes produtos farmacêuticos: carboplatina (Paraplatin, Bristol-Myers Squibb), ciclofosfamida (Genuxal®, Asta Médica); docetaxel (Taxotere $\AA$, Sanofi-Aventis); doxorrubicina (Adriblastina $\AA$, Pfizer); epirrubicina (Farmorrubicina ${ }^{\circledR}, \quad$ Pfizer); fluoruracil (Fluoruracila ${ }^{\circledR}$, Sanofi-Aventis); paclitaxel (Taxol®, Bristol-Myers Squibb); trastuzumabe (Herceptin ${ }^{\circledR}, \quad$ Roche); vinorelbina (Navelbine ${ }$, Asta Médica). Esta lista de preços reflete diferenças em alíquotas de impostos entre os vários estados da federação, sendo aqui considerada a banda intermediária de 17\%".

Os regimes terapêuticos estão descritos na Tabela I. O custo por protocolo de tratamento foi estimado para uma paciente hipotética com superfície corpórea de 1,8 m² (peso: $69 \mathrm{~kg}$, altura: I,7 m), depuração de creatinina estimada de $100 \mathrm{~mL} / \mathrm{min}$ (Cockcroft-Gault), medicamente capaz de receber todos os ciclos de quimioterapia previstos, com intensidade de dose de $100 \%$, sem perda de medicação pelo fracionamento da apresentação farmacêutica.

O estudo FinHER permitia o uso alternativo de docetaxel ou vinorelbina nos ciclos iniciais de tratamento. Assim, a estimativa dos custos para este protocolo foi feita considerando um cenário em que $50 \%$ das mulheres receberiam uma ou outra medicação. 
Tabela I - Estrutura de custos com medicamentos para o tratamento adjuvante completo de uma paciente-tipo (superfície corpórea: I,8 $\mathrm{m}^{2}$ ), segundo diferentes protocolos para câncer de mama HER2-positivo

\begin{tabular}{|c|c|c|c|}
\hline Estudo * & País (ano) & Regimes terapêuticos + & Despesa com medicamentos + \\
\hline NSABP-B3 | e N983| & Estados Unidos (2005) & $\begin{array}{l}\text { AC por quatro ciclos } \mathrm{e} P \text { por quatro ciclos } \\
\mathrm{AC} \text { por quatro ciclos, } \mathrm{P} \text { por quatro ciclos e trastuzumabe por um ano }\end{array}$ & $\begin{array}{l}R \$ 33.980,78 \\
R \$ 214.598,68\end{array}$ \\
\hline HERA & Multinacional (2005) & $\begin{array}{l}\mathrm{AC} \text { por quatro ciclos } \mathrm{e} \text { P por quatro ciclos ou } \mathrm{FEC} \text { por seis ciclos } \\
\mathrm{AC} \text { por quatro ciclos } \mathrm{e} \text { p por quatro ciclos ou } \mathrm{FEC} \text { por seis ciclos, trastuzumabe por um ano }\end{array}$ & $\begin{array}{l}\mathrm{R} \$ 29.614,28 \\
\mathrm{R} \$ 206.824,30\end{array}$ \\
\hline FinHER & Finlândia(2006) & $\begin{array}{l}\text { D ou } V \text { por três ciclos, FEC por três ciclos } \\
\text { D ou } V \text { e trastuzumabe por três ciclos, FEC por três ciclos }\end{array}$ & $\begin{array}{l}R \$ 18.206,83 \\
R \$ 52.285,68\end{array}$ \\
\hline BCIRG 006 & Multinacional (2007) & $\begin{array}{l}\text { AC por quatro ciclos e } D \text { por quatro ciclos } \\
\text { AC por quatro ciclos e } D \text { por quatro ciclos, trastuzumabe por um ano } \\
\mathrm{Cb} \text { e } \mathrm{D} \text { por seis ciclos, trastuzumabe por um ano }\end{array}$ & $\begin{array}{l}\mathrm{R} \$ 34.478,17 \\
\mathrm{R} \$ 211.688,19 \\
\mathrm{R} \$ 231.207,08\end{array}$ \\
\hline
\end{tabular}

* Referências: NSABP-B3I/N983I: Romond EH e cols [6]; HERA: Piccart-Gebhart MJ e cols [5]; FinHER: Joensuu H e cols [4]; BCIRG 006: Robert N] e cols [7].

+ Medicamentos: D, docetaxel; V, vinorelbina; F, 5-fluoruracil; E, epirubicina; C, ciclofosfamida; A, doxorrubicina; P, paclitaxel, Cb, carboplatina.

+ Calculada pelo preço máximo ao consumidor (17\%) - ANVISA. Valor médio ponderado - protocolo FinHER: I: I entre tratamentos com docetaxel ou vinorelbina; protocolo NSABP-B3 I e N983I:

I:I entre tratamentos com paclitaxel $175 \mathrm{mg} / \mathrm{m} 2$ cada três semanas (NSABP-B3 I) ou paclitaxel 80 mg/m2 semanal (N983 I); protocolo HERA: 3:I entre os regimes AC-P ou FEC.

No estudo HERA não foi pré-definido o esquema de quimioterapia inicial a ser empregado, sendo informado apenas que $68 \%$ das mulheres haviam recebido tratamento com antraciclinas, $26 \%$ receberam antraciclinas com taxanas e 6\% receberam tratamento sem antraciclinas ou taxanas ${ }^{5}$. Tendo em vista estas proporções, o padrão atual de tratamento em nosso meio e recomendações internacionais', para a estimativa de custos pelo protocolo HERA consideramos que $75 \%$ das mulheres receberiam quimioterapia com o esquema FECI00 (5-FU $500 \mathrm{mg} / \mathrm{m} 2$, epirrubicina $100 \mathrm{mg} / \mathrm{m} 2$ e ciclofosfamida $500 \mathrm{mg} / \mathrm{m} 2$ por 6 ciclos) e $25 \%$ o regime AC-P (doxorrubicina $60 \mathrm{mg} / \mathrm{m} 2$ e ciclofosfamida $600 \mathrm{mg} / \mathrm{m} 2$ por quatro ciclos, paclitaxel $175 \mathrm{mg} / \mathrm{m} 2$ por quatro ciclos).

Obtidas as estimativas de custo com medicamentos antineoplásicos para cada protocolo terapêutico, estes foram comparados em termos de despesa para se evitar um caso de recidiva da doença em três anos, calculada como o produto entre o NNT e o respectivo custo com medicamentos em cada protocolo. Esta comparação direta entre o desempenho dos estudos foi possível dado o perfil de risco semelhante entre as mulheres dos grupos controle em cada protocolo ${ }^{3}$ e o tempo de seguimento comum compatível com o desfecho selecionado para esta análise (recidiva em três anos) ${ }^{10,12}$. No entanto, foi realizada ainda uma análise de sensibilidade do custo para evitar uma recidiva, segundo extratos comuns de risco variando entre $10 \%$ e $30 \%$.

\section{Resultados}

O custo com medicamentos por regime terapêutico está descrito na Tabela I. Adotou-se o valor médio ponderado sempre que o estudo original previa esquemas antineoplásicos alternativos, quais sejam: protocolo FinHER: I:I entre tratamentos com docetaxel ou vinorelbina; protocolo NSABP-B3 | e N983 |: I:| entre tratamentos com paclitaxel $175 \mathrm{mg} / \mathrm{m} 2$ cada três semanas (NSABP-B3I) ou paclitaxel $80 \mathrm{mg} / \mathrm{m} 2$ semanal (N983 I); protocolo HERA: 3: I entre os regimes $\mathrm{AC}$-P ou FEC.

A Tabela 2 reúne as medidas de eficácia clínica e suas implicações econômicas para cada forma de tratamento e a Figura I mostra a variação do custo com medicamentos para evitar uma recidiva, por protocolo, segundo estratos de risco comuns.

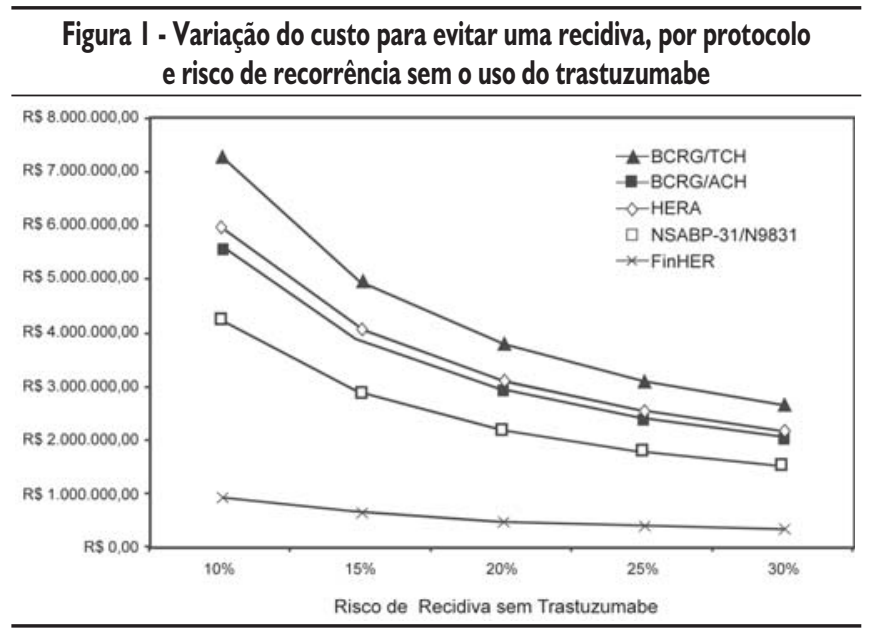

A magnitude do benefício para o uso do trastuzumabe no tratamento adjuvante do câncer de mama HER-2 positivo foi maior nos estudos FinHER e NSABP-B3I/N983I. Em que pese o menor número de pacientes inclúdos no primeiro estudo, o tempo mediano de seguimento por ocasião da publicação superior a 36 meses sugere que seus resultados são definitivos nos termos da medida de eficácia aqui analisada - proporção de pacientes livres de doença após três anos.

O custo com medicamentos para o tratamento individual, segundo o protocolo FinHER, seria quatro vezes menor que empregando algum dos demais protocolos terapêuticos. Por conseguinte, as despesas com medicamentos no mercado de saúde suplementar para se evitar um caso de recidiva do câncer de mama HER-2 positivo seria de quatro a nove vezes menor com o protocolo FinHER, quando comparado com os demais regimes. Esta vantagem para o protocolo FinHER permanece mesmo se considerarmos uma estimativa de benefício mais conservadora, como o limite superior do intervalo de confiança de $95 \%$ para a medida de eficácia. Notamos que estas diferenças seriam ainda maiores para cenários de tratamento em que o risco de recidiva fosse menor que $20 \%$. 
Tabela 2 - Eficácia clínica e custos com medicamentos para diferentes protocolos de quimioterapia adjuvante no câncer de mama HER2-positivo

\begin{tabular}{|c|c|c|c|c|c|}
\hline & \multirow[t]{2}{*}{ FinHER } & \multirow[t]{2}{*}{ NSABP-31/ N983I } & \multirow[t]{2}{*}{ HERA } & \multicolumn{2}{|c|}{ BCIRG-006 } \\
\hline & & & & AC-DT & $\mathrm{CbDT}$ \\
\hline Recidiva em três anos nos controles & $23 \%$ & $25 \%$ & $26 \%$ & $19 \%$ & $19 \%$ \\
\hline Tempo de seguimento (mediana) & 3 anos & 2 anos & 2 anos & 3 anos & 3 anos \\
\hline \multicolumn{6}{|l|}{ Redução de risco } \\
\hline Relativa (IC 95\%) & $52 \%(11 \%-74 \%)$ & $36 \%(20 \%-49 \%)$ & $25 \%(\mid 4 \%-33 \%)$ & $32 \%(17 \%-44 \%)$ & $26 \%(11 \%-39 \%)$ \\
\hline Número necessário para tratar (IC 95\%) & $8(3$ a 28$)$ & $8(7 a \mid 1)$ & $12(9$ a 18$)$ & |4 (I | a 24) & $17(12$ a 34$)$ \\
\hline Custo por tratamento (R\$) & $52.285,68$ & $214.598,68$ & $206.824,30$ & $211.688,19$ & $231.207,08$ \\
\hline Custo para evitar uma recidiva (R\$) & $418.285,44$ & $1.716 .789,44$ & $2.481 .891,58$ & $2.963 .634,62$ & $3.930 .520,43$ \\
\hline Mínimo (limite inferior, 95\%) & $156.857,04$ & $1.502 .190,76$ & $1.861 .418,69$ & $2.328 .570,06$ & $2.774 .485,01$ \\
\hline
\end{tabular}

* Razão de risco para sobrevida livre de doença após 3 anos e intervalo de confiança (IC) de 95\%

\section{Discussão}

A adoção do trastuzumabe no tratamento adjuvante de mulheres com câncer de mama HER2/neu positivo está baseada em sólidas evidências cientíicas. Pelo menos cinco ensaios clínicos randomizados com desfecho e magnitude de efeito clinicamente relevantes demonstraram que o uso deste medicamento promove uma redução no risco de recidiva da ordem de $25 \%$ a $52 \%$ nestas pacientes ${ }^{2}$. Sendo o câncer de mama um dos tumores malignos mais comuns na população feminina, com uma incidência no Brasil estimada pelo Instituto Nacional do Câncer aproximando-se de 50 mil casos anuais ${ }^{13}$, a questão que se impõe é saber qual o modo mais eficiente para os sistemas de saúde incorporarem esta nova tecnologia no tratamento de um quarto destas mulheres, potenciais candidatas ao uso do trastuzumabe.

Há uma preocupação mundial sobre como assegurar do ponto de vista econômico acesso à quimioterapia adjuvante baseada em trastuzumabe para mulheres com câncer de mama HER2/neu-positivo ${ }^{14-16}$. No entanto, as atenções têm se voltado quase que exclusivamente para a estratégia mais divulgada, tóxica e dispendiosa de tratamento - a monoterapia adjuvante prolongada. Neste trabalho demonstramos que restringir o uso do trastzumabe à fase inicial de quimioterapia adjuvante (FinHER) permitiria, do ponto de vista econômico beneficiar até nove vezes mais pacientes que a monoterapia por um ano como empregada nos demais protocolos. Para esta comparação, optou-se por traduzir em termos econômicos o significado do número necessário para tratar para a prevenção de um único desfecho clinicamente significativo: a recidiva do câncer de mama após três anos. A despesa com medicamentos para se evitar um único caso de recidiva situaria se entre pouco mais de $R \$ 4 \mid 8$ mil, no cenário mais favorável (trastuzumabe concomitante aos ciclos iniciais de quimioterapia), e de $\mathrm{R} \$ 1,7$ a 3,9 milhões com trastuzumabe em monoterapia por um ano. As cifras aqui apontadas são muito expressivas para países como o Brasil, onde os recursos para a saúde são escassos, o acesso à mamografia é precário e o diagnóstico do câncer de mama é tardio ${ }^{17-19}$.
Há limitações a serem observadas ao analisar estes resultados. Nos cenários adotados, a quimioterapia adjuvante foi empregada em dose plena durante todo o tratamento, o que não é esperado na prática diária, quando há redução ou omissão de doses devido a efeitos adversos ou fatores supervenientes. Mesmo entre participantes de ensaios clínicos, 7\% (N983I) e II\% (FinHER) das mulheres não receberam todos os ciclos previstos, sendo a monoterapia com trastuzumabe interrompida em 8\% (HERA) e $31 \%$ (N983I) principalmente por redução da fração de ejeção cardíaca. Por sua vez, nestes cenários hipotéticos, as estimativas limitaram-se à contabilização de despesas com agentes antineoplásicos. Não foram estimados custos diretos e indiretos com absenteísmo, honorários e taxas, exames específicos para pesquisa da superexpressão de HER-2/neu, avaliação e monitoramento cardíaco, manipulação e administração dos agentes antineoplásicos, e o acompanhamento e tratamento de efeitos adversos.

Com o custo crescente para o desenvolvimento de novas drogas se refletindo nos altos preços com que são registrados os fármacos inovadores em todo o mundo, a implementação de novas intervenções médicas vem sendo objeto de estudos farmacoeconômicos de custoefetividade?. Nos Estados Unidos, o custo adicional oferecido pelo tratamento adjuvante com trastuzumabe, como proposto no estudo N983I, é comparável a intervenções médicas como a quimioterapia convencional para mulheres acima de 65 anos com câncer de mama e axila positiva, ou a dissecção axilar para mulheres na pós-menopausa com câncer de mama com presença de receptores de estrógeno ${ }^{20}$.

Recentemente, foi avaliado o custo-efetividade para o tratamento adjuvante prolongado com trastuzumabe segundo o protocolo HERA, na Itália e Estados Unidos ${ }^{21}$. Estimou-se que o custo adicional por anos de vida ajustados por qualidade de vida ("QALY") seria de |4.86| euros/"QALY", valor equiparável ao encontrado para outros procedimentos médicos aceitáveis pelo sistema de saúde local, o que justificaria a alocação de recursos para esta nova modalidade terapêutica. No 
entanto, um aspecto do estudo não esclarecido na publicação original pode ter contribuído para uma redução na estimativa dos custos com o uso do trastuzumabe. Estabeleceu-se que o tratamento das recidivas em mulheres que receberam trastuzumabe adjuvante ocorreria apenas com regimes de quimioterapia sem este medicamento (tratamentos com custo estimado em 30.000 euros), enquanto aquelas que não receberam anteriormente a droga seriam tratadas com esquemas contendo trastuzumabe (tratamentos com custo estimado em 50.000 euros). Esta conduta não é observada na prática clínica, na qual se emprega largamente quimioterapia paliativa com trastuzumabe para mulheres com câncer de mama HER2/neu positivo recidivado, independente do tratamento adjuvante que tenham recebido ${ }^{22,23}$.

Antecipa-se formidável desafio aos gestores na área de saúde e à classe médica. No setor público, como conciliar a demanda de cerca de 12 mil mulheres/ano com câncer de mama HER-2/neu positivo e os recursos orçamentários disponíveis, já insuficientes para prover amplo acesso aos serviços da rede assistencial? No setor privado, há que se mediar conflitos de interesses, pois o faturamento de prestadores de serviços de saúde está diretamente atrelado aos custos totais dos procedimentos médicos.

Assim, entendemos que a classe médica e a sociedade devam estar atentas não apenas a critérios de elegibilidade ou diferenciais de eficácia entre os vários protocolos terapêuticos em uso, mas também às implicações econômicas de decisões terapêuticas individuais para o sistema de saúde. Visando maximizar a utilidade dos recursos médicos e societais disponíveis, o uso do trastuzumabe por nove semanas na quimioterapia adjuvante desponta como uma modalidade terapêutica a ser privilegiada em nosso meio como alternativa ética e socialmente responsável de conferirmos uma maior chance de cura para mulheres com câncer de mama HER2/ neu positivo de alto-risco.

Conflito de interesse: $\bigcirc$ autor S.J.M é consultor para incorporação de novas tecnologias em oncologia na Secretaria de Administração do Estado da Bahia (PLANSERV) e Secretaria de Saúde do Estado da Bahia.

\section{SUMMARY}

\section{Clinical and economic issues in adjuvant Chemotherapy FOR HER-2 POSITIVE BREAST CANCER}

PURPOSE. To examine efficacyfigures and drug expenditure for adjuvant chemotherapy in human epidermal growth factor receptor 2 (HER-2) positive breast cancer, in the Brazilian supplemental health insurance market.

METHODS. We obtained efficacy data (disease free survival at 3-years) and drug cost estimate for current adjuvant strategies in HER-2 positive breast cancer: Finland Herceptin (FinHER), National Surgical Adjuvant Breast and Bowel Project(NSAPB-31), North Central Cancer Treatment Group (N9831), Herceptin Adjuvant (HERA) and Breast Cancer International Research Group (BCIRG-006). We estimated clinical impact measures - number needed to treat (NNT) and absolute risk reduction (ARR) - and total drug cost by protocol to avoid one single cancer recurrence.
RESULTS. The largest ARR was $11.7 \%$ (95\% Cl:2.2\% to 21.2\%) in the FinHERstudy, and the smallest in the nonanthracycline arm of the BCIRG trial, $4.9 \%$ (95\% Cl: $1.8 \%$ to 8.1\%). The NNT was 8 (95\% Cl: 3 to 28) in the FinHER, 8 (95\% Cl: 7 to II) in the NSABP-3I/N983I, 12 (95\% Cl: 9 to /8) in the HERA, 14 (95\% Cl: / to 24) in the BCIRG/ Anthracycline, and 17 (Cl 95\% Cl: 12 to 34$)$ in the BCIRG) Nonanthracycline. Drugcost to avoid one single cancer recurrence would be $R \$ 418,285.44$ with the FinHER regimen, $R \$ 1,716,789.44$ with the NSABP-3I/N983I, R\$2,481,891.58 with the HERA, R\$2,963,634.62 with the $B C I R G /$ Anthracicline, and $R \$ 3,930,520.43$ with the $B C I R G /$ Nonanthracycline (exchange rate: $R \$ 1.00=$ USD 0.56).

CONCLUSION. From an economic viewpoint, four to seven times more patients could benefit by using a short-course of trastuzumab at the initial adjuvant chemotherapy cycles (FinHER regimen) than by the prolonged trastuzumab administration as used in other adjuvant schedules. [Rev Assoc Med Bras 2008; 54(6): 494-9]

KEY WORDS: Cost efficiency analysis. Health expenditures. Breast cancer. Adjuvant chemotherapy. Supplemental health. Trastuzumab.

\section{REFERÊNCIAS}

I. Carlson RW, Brown E, Burstein HJ, Gradishar WJ, Hudis CA, Loprinzi C, et al. NCCN Task Force Report: adjuvant therapy for breast cancer. J Natl Compr Cancer Netw. 2006;4(Suppl I):SI-26.

2. Piccart-Gebhart MJ. Adjuvant trastuzumab therapy for HER2overexpressing breast cancer: what we know and what we still need to learn. Eur J Cancer. 2006;42: I7I5-9.

3. Viani GA, Afonso SL, Stefano EJ, De Fendi LI, Soares FV. Adjuvant trastuzumab in the treatment of her-2-positive early breast cancer: a meta-analysis of published randomized trials. BMC Cancer. 2007;7:153

4. Joensuu H, Kellokumpu-Lehtinen PL, Bono P, Alanko T, Kataja V, Asola R, et al. Adjuvant docetaxel or vinorelbine with or without trastuzumab for breast cancer. N Engl J Med. 2006;354:809-20.

5. Piccart-Gebhart MJ, Procter M, Leyland-Jones B, Goldhirsch A, Untch M, Smith I, et al. Trastuzumab after adjuvant chemotherapy in HER2-positive breast cancer. N Engl J Med. 2005;353:1659-72.

6. Romond EH, Perez EA, Bryant J, Suman VJ, Geyer CE, Jr., Davidson NE, et al. Trastuzumab plus adjuvant chemotherapy for operable HER2positive breast cancer. N Engl J Med. 2005;353:1673-84.

7. Robert NJ, Eiermann W, Pienkowski T, Crown J, Martin M, Pawlicki $M$, et al. BCIRG 006: Docetaxel and trastuzumab-based regimens improve DFS and OS over AC-T in node positive and high risk node negative HER2 positive early breast cancer patients: Quality of life (QOL) at 36 months follow-up. ASCO Annual Meeting. 2007. p. 1964-7.

8. Smith I, Procter M, Gelber RD, Guillaume S, Feyereislova A, Dowsett M, et al. 2-year follow-up of trastuzumab after adjuvant chemotherapy in HER2-positive breast cancer: a randomised controlled trial. Lancet. 2007;369:29-36.

9. Earle CC, Chapman RH, Baker CS, Bell CM, Stone PW, Sandberg EA, et al. Systematic overview of cost-utility assessments in oncology. J Clin Oncol. 2000; | 8:3302- 17.

10. Altman DG ,Andersen PK. Calculating the number needed to treat for trials where the outcome is time to an event. BMJ. 1999;3 |9: | 492-5.

I I. Sanitária CdRdMdM-ANdV. Listas de preços máximos de medicamentos ao consumidor; 2007 [citado dez 2007]. Available from: http:// www.anvisa.gov.br/monitora/precos.htm.

12. Sharp SJ, Thompson SG ,Altman DG. The relation between treatment benefit and underlying risk in meta-analysis. BMJ. 1996;313:735-8. 
13. Saúde. INdC-Md. Estimativa 2006: incidência de câncer no Brasil[citado dez 2007]. Available from: http://www.inca.gov.br/ estimativa/2006.

14. Barrett A, Roques T, Small M, Smith RD. How much will Herceptin really cost? BMJ. 2006;333: I I 18-20.

15. Hillner BE,Smith TJ. Do the large benefits justify the large costs of adjuvant breast cancer trastuzumab? J Clin Oncol. 2007;25:611-3.

16. Norum J. The cost-effectiveness issue of adjuvant trastuzumab in early breast cancer. Expert Opin Pharmacother. 2006;7:1617-25.

17. De Moraes AB, Zanini RR, Turchiello MS, Riboldi J ,De Medeiros LR. Estudo da sobrevida de pacientes com câncer de mama atendidas no hospital da Universidade Federal de Santa Maria, Rio Grande do Sul, Brasil. Cad Saude Publica. 2006; 22:2219-28.

18. Koch H, Peixoto J ,Eiras A. Análise da infra-estrutura para a mamografia no Brasil. Radiol Bras. 2000;33:23-30.

19. Mendonca GA, Silva AM, Caula WM. Características tumorais e sobrevida de cinco anos em pacientes com câncer de mama admitidas no Instituto Nacional de Câncer, Rio de Janeiro, Brasil. Cad Saude Publica. 2004;20: 1232-9.
20. Kurian AW, Thompson RN, Gaw AF, Arai S, Ortiz R, Garber AM. A costeffectiveness analysis of adjuvant trastuzumab regimens in early HER2/ neu-positive breast cancer. J Clin Oncol. 2007;25:634-4I .

21. Liberato NL, Marchetti M ,Barosi G. Cost effectiveness of adjuvant trastuzumab in human epidermal growth factor receptor 2-positive breast cancer. J Clin Oncol. 2007;25:625-33.

22. Garcia-Saenz JA, Martin M, Puente J, Lopez-Tarruella S, Casado A, Moreno F, et al. Trastuzumab associated with successive cytotoxic therapies beyond disease progression in metastatic breast cancer. Clin Breast Cancer. 2005;6:325-9.

23. Pusztai $L$,Esteva FJ. Continued use of trastuzumab (herceptin) after progression on prior trastuzumab therapy in HER-2-positive metastatic breast cancer. Cancer Invest. 2006;24: 187-91.

Artigo recebido: 19// I/07 Aceito para publicação: 28/01/08 